CAPÍTULO 1

\title{
Avaliação de compostos voláteis em vinhos produzidos com uvas "Niágara Branca" (Vitis labrusca) irradiadas com UV-C
}

Simara Somacal, Rodrigo Valvassori Gindri, Fernanda Wouters Franco, Sabrina Somacal, Cláudia Kaehler Sauter, Fabrízio da Fonseca Barbosa

https://doi.org/10.4322/mp.978-65-991393-3-8.c1

\section{Resumo}

A uva "Niágara Branca" é uma das variedades mais cultivadas no estado do Rio Grande do Sul para a produção de vinhos brancos de mesa. O aroma final de um vinho é formado por um grande número de compostos químicos voláteis, presentes em concentrações variáveis e detectáveis pelo sistema olfativo humano. A radiação ultravioleta do tipo C (UV-C) é um elicitor abiótico capaz de causar pequenos danos em tecidos vegetais, que faz com que as plantas produzam fitoalexinas como mecanismo de defesa, podendo modificar a composição de compostos voláteis desses vegetais. O objetivo deste estudo foi avaliar o teor de compostos voláteis em vinhos produzidos com uvas "Niágara Branca" expostas à UV-C. Os compostos voláteis foram analisados em cromatógrafo a gás acoplado a espectrômetro de massas. Foram quantificados 54 compostos voláteis, sendo os ésteres o grupo químico que apresentou o maior aumento de sua concentração $(35,7 \%)$ nos vinhos produzidos com uvas previamente irradiadas com UV-C, com destaque para o 9-decenoato de etila que teve um aumento de $135,7 \%$. Os resultados obtidos no presente estudo permitem concluir que a irradiação de uvas "Niágara Branca" com UV-C gera uma resposta fisiológica que aumenta a concentração de compostos voláteis.

Palavras-chave: aroma, elicitor abiótico, hormese, Niágara, vinhos de mesa.

\section{Introdução}

A produção de uvas no Brasil está localizada principalmente na região Sul do país. Constitui-se em atividade consolidada, com importância socioeconômica, principalmente nos Estados do Rio Grande do Sul e de Santa Catarina, os quais respondem por $73 \%$ da produção nacional de vinhos. A uva Niágara Branca é uma das principais cultivares de Vitis labrusca, e juntamente com a uva Isabel, são destaques como "uvas comuns", sendo variedades rústicas e amplamente utilizadas na fabricação de vinhos de mesa. Em 2015, esses mostraram aumento de 7,21\% na sua produção, com alta de $7,63 \%$ para os tintos e de $5,66 \%$ para os brancos (MELLO, 2015).

A cor, o sabor, o aroma e a adstringência são atributos importantes para definir a qualidade de um vinho (RIBÉREAU-GAYON et al., 2006), podemos destacar o aroma como sendo um dos principais aspectos sensoriais levados em consideração pelos consumidores, sendo diversas as substâncias que compõem os compostos voláteis do vinho e que contribuem para a formação do buquê do mesmo (JIANG et al., 2013; NIU 
et al., 2011). Além dos aromas provenientes das uvas, chamados "varietais", a fração volátil dos vinhos advém também da fermentação por leveduras e outros microorganismos, envolvendo uma série de reações bioquímicas e, também, o envelhecimento da bebida, tanto em barricas de carvalho como na garrafa (BURIN et al., 20011; MAURIELLO et al., 2009). Atualmente, já foram identificados mais de 800 compostos voláteis em vinhos e sabe-se que somente algumas dezenas são aromas ativos para finalidades de diferenciação (ou seja, estão presentes em concentração acima do limiar de detecção olfativa em vinhos). Esses compostos pertencem a diversas classes químicas e estão presentes em concentrações que vão desde nanogramas a miligramas por litro (BAYONOVE, 2000; FALCÃO et al., 2007).

Hormese é um estímulo de uma resposta positiva por baixas doses de qualquer agente potencialmente nocivo. Um tipo de tratamento pós-colheita, com efeito, hormético é o uso de baixas doses de radiação UV-C, que atua como elicitor, causando danos que ativam mecanismos de reparo aos efeitos da irradiação, estimulando a uva a produzir fitoalexinas, que são metabólitos secundários produzidos pelas plantas em resposta a esse estresse (GOMEZ et al., 2010; LAMIKANRA; RICHARD; PARKER, 2002).

Portanto o objetivo deste estudo foi avaliar produção de vinhos a partir de uvas Niágara Branca submetidos a radiação UV-C.

\section{Material e Métodos}

\subsection{Material}

O experimento foi realizado com uvas Vitis labrusca da cultivar 'Niágara Branca' (aproximadamente $40 \mathrm{~kg}$ ), provenientes da Vinícola Velho Amâncio (Santa Maria/RS), colhidas em fevereiro de 2011. Após a toalete, as uvas foram divididas em 2 grupos experimentais: controle (dose de $0 \mathrm{~kJ} \cdot \mathrm{m}^{-2}$ ) e irradiadas (dose $3 \mathrm{~kJ} . \mathrm{m}^{-2}$ ), com três repetições em cada grupo experimental.

\subsection{Métodos}

\subsubsection{Irradiação ultravioleta do tipo C}

O método de irradiação foi empregado conforme Sautter et al. (2008). As unidades amostrais foram submetidas a irradiação em uma câmara anti-reflectiva, constituída por fonte ultravioleta com emissão na região do UV-C (marca Phillips ${ }^{\circledR}$ - modelo TUV15W/ G15T8 Longe Life, potência de $15 \mathrm{~W}$ ) e expostas a uma irradiância de 3,048 W. $\mathrm{m}^{2}$ e comprimento de onda de $254 \mathrm{~nm}$ (parâmetros previamente aferidos pela empresa Ensoltec ${ }^{\circledR}$, Porto Alegre). O tempo de exposição das uvas foi de 16,4 min, determinado segundo Diffey (2002) pela relação da dose e irradiância emitida pela lâmpada ultravioleta. Posteriormente, todas as uvas (controle e irradiada) foram mantidas em câmara com controle de temperatura a $20^{\circ} \mathrm{C}$ por três dias, para que houvesse uma resposta fisiológica à irradiação UV-C.

\subsubsection{Vinificação}

A microvinificação foi conduzida conforme Miele e Rizzon (2006) com algumas adaptações. As uvas foram desengaçadas e esmagadas manualmente e o mosto foi acondicionado em microvinificadores de polietileno e adicionada enzima pectinolítica (pectinoesterase) LAFAZYM ${ }^{\circledR} \mathrm{CL}$, marca Laffort $^{\circledR}\left(1 \mathrm{~g} \cdot \mathrm{L}^{-1}\right)$. Após $4 \mathrm{~h}$ foi realizada a descuba e adicionada a levedura Saccharomyces cereviseae, ZYMAFLORE FX10 ${ }^{\circledR}$ e 0 
ativante Bioactiv ${ }^{\circledR}$, marca Laffort ${ }^{\circledR}$, conforme as especificações do fabricante. A fermentação foi conduzida durante 12 dias em temperatura de $16{ }^{\circ} \mathrm{C}$ no laboratório de fermentações no núcleo integrado de desenvolvimento de análises laboratoriais (NIDAL/UFSM). Diariamente foram retiradas alíquotas do mosto para verificar a concentração de sólidos solúveis totais (SST) e a dinâmica da fermentação. A fermentação foi finalização quando os SST se mantiveram constantes por 2 dias e inferiores a 4 Brix. A mensuração dos SST foi feita com refratômetro portátil (modelo REF 103, Biobrix ${ }^{\circledR}$ ) conforme Instituto Adolfo Lutz (2008).

Após este período, os vinhos foram clarificados (bentonite) e sulfitados (60 ppm de $\mathrm{SO}_{2}$ ) por um período de 2 semanas. Posteriormente, os vinhos foram trafegados e submetidos à temperatura de $1{ }^{\circ} \mathrm{C}$ por 6 meses, em câmara refrigerada para que ocorresse a estabilização tartárica. Passado este período de estabilização, as amostras foram acondicionadas em garrafas de vinho de cor verde com capacidade de $375 \mathrm{~mL}$ e tapadas com rolhas de corticeira. As análises cromatográficas foram realizadas após 6 meses do engarrafamento dos vinhos.

\subsubsection{Análise de compostos voláteis}

A extração das amostras para as análises cromatográficas gasosas tanto qualitativas quanto quantitativas, foi por microextração em fase sólida (SPME), realizada seguindo o método proposto por Tao e Zhang (2010) com adição de padrão interno 3-octanol $\left(4,1 \mu \mathrm{g} \cdot \mathrm{mL}^{-1}\right)$ e utilizando a fibra DVB/Car/PDMS (divinilbenzeno/carboxen/ polidimetilsiloxano, Sulpelco, 50/30 $\mu \mathrm{m} \times 20 \mathrm{~mm}$, Supelco), que foi exposta por $30 \mathrm{~min}$ ao Headspace. A identificação dos compostos voláteis foi feita utilizando cromatógrafo gasoso Shimadzu GC $2010^{\circledR}$, acoplado a espectrômetro de massas (Shimadzu QP 2010 Plus $\left.^{\circledR}\right)$ utilizando uma coluna capilar de polietilenoglicol CP-WAX 52 CB $^{\circledR}(30,0 \mathrm{~m}$ $\times 0,25 \mathrm{~mm} \times 0,25 \mu \mathrm{m}$ ). O programa de temperatura da coluna foi iniciado a $35{ }^{\circ} \mathrm{C}$, permanecendo por $1 \mathrm{~min}$, e posteriormente aumentando até $240{ }^{\circ} \mathrm{C}$ a uma taxa de $4^{\circ} \mathrm{C} \cdot \mathrm{min}^{-1}$, temperatura que foi mantida por $10 \mathrm{~min}$. $\mathrm{O}$ gás de arraste foi o hélio, tendo uma vazão de $2,8 \mathrm{~mL} \cdot \mathrm{min}^{-1}$ permanecendo à pressão constante de $61,8 \mathrm{kPa}$. A temperatura da interface no GC/MS e da fonte de íons foi de $250^{\circ} \mathrm{C}$. A ionização dos compostos foi configurada no modo de ionização por elétrons a $70 \mathrm{eV}$. O analisador de massas quadrupolos monitorando as massas de íons operou no modo de varredura de 40 a $350 \mathrm{~m} \cdot \mathrm{z}^{-1}$. A voltagem aplicada ao detector foi de $0,8 \mathrm{kV}$. Na identificação dos compostos voláteis foi utilizada a série de alcanos $\mathrm{C}_{6}-\mathrm{C}_{22}$, comparando o espectro de massas com a biblioteca NIST e a confirmação destes dados foi feita por comparação entre os Índices de Kovats (IK) ou Índices de Retenção (IR) dos compostos identificados pelo detector, com os citados pela literatura ou com IK e IR obtidos a partir de análises de padrões.

A quantificação dos compostos voláteis foi realizada em cromatógrafo a gás VARIAN ${ }^{\circledR}$, modelo Star 3400 cx, com detector de ionização de chama (GC/FID). O método de extração e as condições das análises cromatográficas foram semelhantes as citadas anteriormente, diferindo-se pelo fato de o gás de arraste ser o hidrogênio em vazão de 2,8 $\mathrm{mL} \cdot \mathrm{min}^{-1}$ e pressão constante de $61,8 \mathrm{kPa}$. Nesta análise também foram aplicadas as séries de alcano C6-C22 para pareação através do IK dos analitos identificados no CG. Os resultados foram expressos em equivalentes $\mu \mathrm{g}$ de 3 octanol. $\mathrm{mL}^{-1}$. 


\subsubsection{Análise estatística}

Após a verificação da normalidade dos dados, as médias do grupo controle versus médias do grupo irradiado foram comparadas pelo teste t de Student a um nível de 5\% de significância, utilizando o programa Statistic versão 9.0.

\section{Resultados e Discussão}

As concentrações médias dos compostos voláteis presentes no vinho, produzidos a partir de uvas Niágara Branca irradiadas e não irradiadas com UV-C, se encontram na Tabela 1 divididas por classes químicas, em equivalentes microgramas de 3octanol. $\mathrm{mL}^{-1}$ de vinho.

Tabela 1. Concentração de compostos voláteis $\left(\mu \mathrm{g} \cdot \mathrm{mL}^{-1}\right)$ obtidos no vinho da cultivar "Niágara Branca" por tratamentos.

\begin{tabular}{|c|c|c|c|}
\hline \multirow[b]{2}{*}{ Grupos } & \multirow[b]{2}{*}{$\mathrm{IK}^{\star *}$} & \multicolumn{2}{|c|}{ Dose UV-C } \\
\hline & & $0 \mathrm{KJ} \cdot \mathrm{m}^{-2}$ & $3 \mathrm{KJ} \cdot \mathrm{m}^{-2}$ \\
\hline \multicolumn{4}{|l|}{ Ácidos } \\
\hline Ácido acético & 1457 & $1,74 \pm 0,39$ & $2,43 \pm 0,45$ \\
\hline Ácido 2-metil-propanoico & 1564 & $0,97 \pm 0,12$ & $1,00 \pm 0,24$ \\
\hline Ácido butírico & 1634 & $0,31 \pm 0,01$ & $0,3 \pm 0,03$ \\
\hline Ácido hexanoico & 1854 & $5,75 \pm 0,64$ & $5,60 \pm 0,08$ \\
\hline Ácido 2-etil hexanoico & 1931 & $0,39 \pm 0,01^{*}$ & $0,26 \pm 0,01^{*}$ \\
\hline Ácido octanoico & 2074 & $12,56 \pm 2,52$ & $12,89 \pm 0,61$ \\
\hline$\Sigma$ & & 21,72 & 22,48 \\
\hline \multicolumn{4}{|l|}{ Álcoois } \\
\hline Isobutanol & 1113 & $27,16 \pm 5,76$ & $30,33 \pm 4,93$ \\
\hline Álcool isoamílico & 1236 & $37,97 \pm 0,06^{*}$ & $43,55 \pm 1,18^{*}$ \\
\hline 3-metil-1-pentanol & 1327 & $0,86 \pm 012$ & $0,70 \pm 0,12$ \\
\hline 1-hexanol & 1355 & $13,67 \pm 1,29$ & $11,10 \pm 1,39$ \\
\hline Cis-3-Hexenol & 1394 & $4,02 \pm 0,04$ & $4,10 \pm 0,01$ \\
\hline 3-Octenol & 1447 & $0,96 \pm 0,03$ & $0,88 \pm 0,17$ \\
\hline 2-etil-1-hexanol & 1489 & $0,74 \pm 0,08^{*}$ & $0,32 \pm 0,07^{\star}$ \\
\hline 2,3-butanodiol & 1544 & $1,64 \pm 0,12$ & $1,31 \pm 0,10$ \\
\hline 1-Octanol & 1551 & $0,83 \pm 0,07$ & $0,78 \pm 0,10$ \\
\hline 3-Terpinenol & 1580 & $0,07 \pm 0,02$ & $0,11 \pm 0,09$ \\
\hline
\end{tabular}


2,3-Butanediol

Propilenoglicol

1-Nonanol

Metionol

Álcool Feniletílico

n-Dodecanol

$\Sigma$

Aldeidos

1,4 butanediol hexadecanal acetal

$\Sigma$

\section{Cetonas}

2-Heptanona

Metil heptanona

$\Sigma$

\section{Ésteres}

Acetato de isobutila

Butanoato de etila

2-Metilbutanoato de etila

Isovalerato de etila

Acetato de isoamila

trans-Crotonato de etila

4-metil pentanoato de metila

Hexanoato de etila

2-Hexenoato de etila

Octanoato de etila

Lactato de isoamila

2- Furoato de etila

Decanoato de etila
1584

1604

1667

1725

1924

1964

1159

1191

1332

1023

1044

1061

1077

1129

1167

1188

1221

1340

1434

1571

1638

1645

$$
0,38 \pm 0,09
$$

$0,58 \pm 0,24$

$0,28 \pm 0,06$

$0,38 \pm 0,31$

$0,62 \pm 0,23$

$0,21 \pm 0,01$

$0,79 \pm 0,03^{*}$

$1,37 \pm 0,11^{*}$

$110,48 \pm 11,19$

$130,66 \pm 9,24$

$0,38 \pm 0,02$

$0,34 \pm 0,07$

200,47

226,72
$0,22 \pm 0,07$

0,18

0,22

$$
\begin{array}{cc}
0,28 \pm 0,12 & 0,36 \pm 0,24 \\
0,47 \pm 0,27^{*} & 0,05 \pm 0,01^{*} \\
0,75 & 0,41
\end{array}
$$


Benzoato de etila

Succinato de dietila

9-Decenoato de etila

Glutarato de dietila

Acetato de feniletila

3-metil butil butenoato de etila

$\Sigma$

Éter

Metil2(Z)pentenil eter

1019

1,1-Dietoxibutano-3 metil

1-Etoxietoxi-1- pentano

1,2 Epoxiciclodecano

$\Sigma$

Hidrocarbonetos

Estireno

1256

$\Sigma$

\section{Terpenos}

Óxido de nerol

1464

Hotrienol

1616

$\alpha$-Terpinenol

Citronelol

$\Sigma$

Fenol

4- etil guaiacol

2015

1081

1106

1623

1705

1772

$$
\begin{array}{cc}
0,48 \pm 0,06 & 0,60 \pm 0,15 \\
10,79 \pm 0,67^{*} & 13,54 \pm 0,10^{*} \\
0,14 \pm 0,04^{*} & 0,33 \pm 0,06^{*} \\
0,35 \pm 0,05 & 0,17 \pm 0,06 \\
2,09 \pm 0,05^{*} & 2,48 \pm 0,04^{*} \\
0,63 \pm 0,01^{*} & 0,85 \pm 0,01^{*} \\
612,97 & 698,02
\end{array}
$$

$0,19 \pm 0,02$

$0,19 \pm 0,03$

$0,07 \pm 0,01$

$0,07 \pm 0,02$

$0,18 \pm 0,03$

$0,21 \pm 0,07$

$0,43 \pm 0,01$

$0,43 \pm 0,03$

0,87

0,9

$0,24 \pm 0,01^{*}$

$0,07 \pm 0,01^{*}$

0,67

0,5

$\Sigma$
$0,26 \pm 0,04$

$0,17 \pm 0,02$

$0,17 \pm 0,01$

$7,06 \pm 5,89$

$4,08 \pm 1,09$

$0,39 \pm 0,02$

$0,40 \pm 0,06$

4,89

7,88

$0,91 \pm 0,18^{*} \quad 0,26 \pm 0,03^{*}$

0,91

0,26

**índices de Kovats comparados com obtidos por padrões e pela literatura em: ALVES; FRANCO, 2003; ANTALICK; PERELLO; REVEL, 2010; COMUZZO, et al, 2006; GARRUTI et al., 2006; NIU et al., 2011; RIU-AUMATELL et al., 2006; TAO; LIU; LI, 2008; SAN-JUAN et al., 2010; XI et al., 2011. Resultados expressos como média \pm erro padrão. *Médias diferem entre si pelo teste de Student-t (linha). 
A partir da Tabela 1 pode-se observar que houve alterações em diversos compostos voláteis, dentro de quase todas as classes químicas. Porém não houve diferença significativa na concentração entre grupos, mas este valor não é absoluto, uma vez que alguns compostos encontrados por cromatografia não puderam ser quantificados por GC-FID, pois estes coeluiram entre si ou com o etanol.

Foram encontrados 89 compostos voláteis presentes no vinho, dos quais 54 foram identificados e quantificados, sendo estes: 6 ácidos (11,1\%), 16 álcoois (29,6\%), 1 aldeído (1,9\%), 2 cetonas (3,7\%), 19 ésteres (35,1\%), 4 éteres (7,4\%), 1 hidrocarbonetos (1,9\%), 4 terpenos $(7,1 \%)$ e 1 fenol (1,9\%). Deste total, 14 deles apresentaram alguma diferença significativa $(p<0,05)$ entre os tratamentos. Dos seis ácidos identificados, o ácido 2-etil hexanóico apresentou uma redução significativa de $33 \%$ em relação a dose de $0 \mathrm{KJ} \mathrm{m}^{-2}(p<0,05)$.

Dentre as classes químicas de importância para os aromas dos vinhos, os álcoois superiores são formados principalmente durante a fermentação alcoólica, resultantes da degradação de aminoácidos através do mecanismo de Ehrlich ou catabolismo de açúcares (BOULTON et al., 1996; EBELER, 2001). Entre os dezessete álcoois identificados três apresentaram diferença significativa $(p<0,05)$ na concentração entre os tratamentos. Houve um aumento de $14,7 \%$ no álcool isoamílico e de $73,4 \%$ no metionol e redução de $56,7 \%$ no 2-metil-1-hexanol quando comparado a dose de 3 $\mathrm{KJ} . \mathrm{m}^{-2}$ em relação ao controle.

Dentre as cetonas detectadas houve um decréscimo significativo de $89,4 \%$ na concentração da metil heptanona na dose $3 \mathrm{~kJ} \cdot \mathrm{m}^{-2}$ quando comparada a $0 \mathrm{~kJ} \cdot \mathrm{m}^{-2}$. A irradiação com UV-C não alterou significativamente $(p<0,05)$ nenhum dos compostos pertencentes a classe dos aldeídos, éteres e terpenos quando comparados ao controle. Já em relação à classe dos hidrocarbonetos, a irradiação com UV-C reduziu $70,8 \%$ a concentração do estireno em relação ao controle. A redução deste composto volátil é benéfico ao aroma do vinho pois demostra que, possivelmente, houve uma redução no aroma pungente do vinho, uma vez que esse tipo de aroma está relacionado a esse hidrocarboneto (WELKE, 2012).

Os vinhos produzidos com uvas Niágara Branca contêm 20 ésteres identificados, destes 7 apresentaram diferença significativa entre os tratamentos. Nos vinhos provenientes de uvas tratadas com UV-C na dose de $3 \mathrm{~kJ} \cdot \mathrm{m}^{-2}$ houve um aumento de $135,7 \%$ no 9 -decenoato de etila, $34,9 \%$ no succinato de etil 3-metil butila, $25,5 \%$ no succinato de dietila, $18,6 \%$ no Acetato de feniletila, $17,7 \%$ no hexanoato de etila, em relação ao controle. Os ésteres trans-crotonato de etila e octanoato de etila apresentaram uma redução significativa $(p<0,05)$ de $22,6 \%$ e $7,5 \%$, respectivamente, quando comparado o tratamento $3 \mathrm{~kJ} \cdot \mathrm{m}^{-2}$ de irradiação com UV-C ao controle. A classe química dos ésteres foi a que apresentou um maior aumento na concentração de compostos com a irradiação UV-C.

Nos vinhos provenientes de uvas irradiadas nota-se que dois ésteres derivados do ácido succínico, encontram-se aumentados (succinato de dietila e succinato de etil 3metil butila). Porém, esse ácido não foi mensurado, porque foi coeluído com outros compostos durante a cromatografia gasosa. Ainda, houve o aumento de ésteres formados a partir do álcool isoamílico, que apresentaram concentração média superior nos vinhos produzidos com uvas tratadas com UV-C, no entanto seus ésteres (isovalerato de etila e acetato de isoamila) não se diferiram estatisticamente nos 
vinhos produzidos com uvas tratadas com UV-C do grupo controle. Esse aumento pode ser devido ao aumento da concentração de isoleucina e leucina, aminoácidos precursores desse álcool e ácido (FALQUÉ; FERNÁNDEZ; DUBOURDIEU, 2002; PARESTELLO et al., 2006).

O vinho da cultivar "Niágara Branca" produzido com uvas irradiadas com UV-C na dose de $3 \mathrm{~kJ} \cdot \mathrm{m}^{-2}$ possivelmente apresente notas aromáticas frutais mais pronunciadas que os vinhos controle, pois houve um aumento significativo na concentração de muitos ésteres, como visto na Tabela 1. De acordo com a Tabela 2, que traz dados da literatura referentes às notas aromáticas, a maioria dos compostos que apresentaram aumento significativo de concentração possuem este tipo de aromas. Adicionalmente, houve redução da concentração de compostos voláteis que afetam negativamente 0 aroma de vinhos, como o 4-etil guaiacol e o ácido 2-etil hexanóico, que tiveram, respectivamente, uma redução de $71,4 \%$ e $33 \%$ no grupo irradiado com a UV-C. O 4etil guaiacol quando presente em elevadas concentrações atribui ao vinho aroma de fumaça (CLARKE; BAKKER, 2004) e o ácido 2-etil hexanóico quando em elevadas concentrações confere ao vinho um aroma mais rançoso (gordura) e também pode afetar negativamente o processo fermentativo (CLARKE; BAKKER, 2004). Nossos dados estão de acordo com os estudos de Treptow (2012), que apontam que a irradiação com UV-C em uvas da cultivar "Isabel" intensificaram os compostos voláteis presentes nessa cultivar, sobretudo os ésteres, com destaque para o butanoato de etila (aroma doce), o hexanoato de etila (aroma frutado) e o hexanoato de 2-etila (aroma frutado).

Tabela 2. Notas olfativas de compostos voláteis.

\begin{tabular}{ll}
\hline Composto & Aroma $^{1}$ \\
\hline Ácido 2-etil hexanoico & Gordura, levedura, queijo, ranço $^{(1)}$ \\
2-etil 1-hexanol & Pungente, álcool, malte, queimado, queijo ${ }^{(2,3)}$ \\
Metionol & Herbáceo, pungente ${ }^{(3)}$ \\
Metil heptanona & ------- \\
trans-Crotonato de etila & Cogumelo, vinil, borracha, amadeirado, groselha ${ }^{(2)}$ \\
Octanoato de etila & --------- \\
Hexanoato de etila & Frutado, gordura ${ }^{(3)}$ \\
Succinato de dietila & Frutado, morango, anis, doce ${ }^{(2,3)}$ \\
9-Decenoato de etila & Fruta, vinho, fermentado, floral $^{(1,2)}$ \\
Acetato de feniletila & Frutado $^{(3)}$ \\
Succinato de etil 3-metil butila & Flor de camomila, aipo, frutado, mofado, chá $^{(1)}$ \\
Estireno & Pungente, perfumado, assado $^{(3)}$
\end{tabular}

1 Notas olfativas descritas na literatura. (1) ANTALICK; PERELLO; REVEL, 2010; (2) NIU et al.,2011; (3) TREPTOW (2012). 


\section{Conclusão}

Foram detectados a presença de 56 compostos voláteis no vinho produzido com uvas Niágara Branca. A irradiação com UV-C provocou um aumento de $13,5 \%$ de seus compostos voláteis. Houve um aumento da concentração de 14 compostos voláteis dos 54 presentes nos vinhos Niágara Branca, produzidos com uvas irradiadas. Entre os principais compostos que apresentaram aumento de concentração após o tratamento com a irradiação com UV-C estão o álcool isoamílico, metionol, 9decenoato de etila, succinato de etil 3-metil butila, succinato de dietila, acetato de feniletila, hexanoato de etila. Assim, espera-se dos vinhos irradiados um aroma mais frutado.

\section{Agradecimento}

Esse estudo foi parcialmente financiado pela Fundação de Amparo à Pesquisa do Rio Grande do Sul (FAPERGS).

\section{Referências}

ALVES, G. L.; FRANCO, M. R. Headspace gas chromatography-mass spectrometry of volatile compounds in murici (Byrsonima crassifolia Rich). Journal of Chromatography A, v. 985, p. 297-301. 2003. http://dx.doi.org/10.1016/s00219673(02)01398-5.

ANTALICK, G.; PERELLO, M. C. REVEL, G. Development, validation and application of a specific method for the quantitative determination of wine esters by headspacesolid-phase microextraction-gas chromatography-mass spectrometry. Food $\begin{array}{llllll}\text { Chemistry, } & \text { v. } & 121, & \text { n. } 4, & \text { p. } & 1236-1245,\end{array}$ http://dx.doi.org/10.1016/j.foodchem.2010.01.011.

BAYONOVE, C. Aromas. In: FLANZY, C (Ed.). Enologia, fundamentos científicos y tecnológicos. Madrid: Mundi-Prensa: AMV Ediciones, 2000. 245-30 p.

BOULTON, R.B. et al. Principles and practices of winemaking. Chapman and Hall: New York, p.604, 1996. http://dx.doi.org/10.1007/978-1-4757-6255-6.

BURIN, V. M. et al. Cabernet Sauvignon wines from two different clones, characterization and evolution during bottle ageing. LWT - Food Science and Technology, v.44, n.9, p.1931-1938, 2011. http://dx.doi.org/10.1016/j.Iwt.2011.05.001.

CLARKE, R.J.; BAKKER, J. Wine: flavor chemistry. Chichester: Wiley-Blackwell, 2004. 336p.

COMUZZO, P. et al. Yeast derivatives (extracts and autolysates) in winemaking: Release of volatile compounds and effects on wine aroma volatility. Food Chemistry, v. 99, n.2, p. 217-230, 2006. http://dx.doi.org/10.1016/j.foodchem.2005.06.049.

DIFFEY, L. D. Sources and measurement of ultraviolet radiation. Methods, v.28, p.413, 2002. http://dx.doi.org/10.1016/S1046-2023(02)00204-9. 
EBELER, S. E. Analytical chemistry: unlocking the secrets of wine flavor. Food Reviews International, v.17, n.1, p.45-64, 2001. http://dx.doi.org/10.1081/FRI-100000517.

FALQUÉ, E.; FERNÁNDEZ, E.; DUBOURDIEU D. Volatile Components of Loureira, Dona Branca, and Treixadura Wines. Journal of Agricultural and Food Chemistry, v.50, n.3, p.538-543, 2002. https://doi.org/10.1021/jf010631s.

FALCÃO, L. D. et al. A survey of seasonal temperatures and vineyard altitude influences on 2-methoxy-3-isobutylpyrazine, C13 - norisoprenoids and the sensory profile of brazilian Cabernet Sauvignon wines. Journal of Agricultural and Food Chemistry, v.55, p.3605-3612, 2007. https://doi.org/10.1021/jf070185u.

GARRUTI, D. S. Assessment of aroma impact compounds in a cashew apple-based alcoholic beverage by GC-MS and GC-olfactometry. LWT - Food Science and Technology, v. 39, p. 372-377, 2006. https://doi.org/10.1016/j.Iwt.2005.02.006.

GOMEZ, P. L. et al. Effect of ultraviolet-C light dose on quality of cut-apple: Microorganism, color and compression behavior. Journal of Food Engineering, v. 98, p. 60-70, 2010. https://doi.org/10.1016/j.jfoodeng.2009.12.008.

INSTITUTO ADOLF LUTZ. Métodos físico-químicos para análise de alimentos, 4. ed. São Paulo, 2008. p. 354, 421 - 425, 564.

JIANG, B. et al. Comparison on aroma compounds in Cabernet Sauvignon and Merlot wines from four wine grape-growing regions in China. Food Research International, v.51, p.482-489, 2013. https://doi.org/10.1016/0.1016/j.foodres.2013.01.001.

LAMIKANRA, O.; RICHARD, O. A.; PARKER, A. Ultraviolet induced stress response in fresh cut cantaloupe. Phytochemistry, v.60, n.1, p.27-32, 2002. https://doi.org/10.1016/S0031-9422(02)00048-1.

MAURIELLO, G. et al. SPME-GC method as a tool to differentiate VOC profiles in Saccharomyces cerevisiae wine yeasts. Food Microbiology, v.26, p.246-252, 2009. https://doi.org/10.1016/j.fm.2009.01.003.

MELLO, L.M.R. Desempenho da vitivinicultura brasileira em 2015. Agroindústria Estudos socioeconômicos e ambientais Pesquisa, Desenvolvimento e Inovação. Campo \& Negócio - HF, Uberlândia, v. 8, n. 128, 2016. p. 108-116.

MIELE, A; RIZZON, L. A. Efeito de elevadas produtividades do vinhedo nas características físico-químicas e sensoriais do vinho Merlot. Ciência Rural, v.36, n.1, p.271-278, 2006. https://doi.org/10.1590/S0103-84782006000100042.

NIU, Y. et al. Characterization of odor-active compounds of various cherry wines by gas chromatography-mass spectrometry, gas chromatography-olfactometry and their correlation with sensory attributes. Journal of Chromatography B, v.879, p.22872293, 2011. https://doi.org/10.1016/j.jchromb.2011.06.015.

RIBÉREAU-GAYON, P. et al. Handbook of enology: The chemistry of wine, v.2. Chichester: John Wiley and Sons, 2006. 450p.

RIU-AUMATELL, M. et al. Development of volatile compounds of cava (Spanish sparkling wine) during long ageing time in contact with lees. Food Chemistry, v. 95 p. 237-242, 2006. https://doi.org/10.1016/j.foodchem.2005.01.029. 
SAN-JUAN, F. Producing headspace extracts for the gas chromatographyolfactometric evaluation of wine aroma. Food Chemistry, v. 123, p. 188-195, 2010. https://doi.org/10.1016/j.foodchem.2010.03.129.

SAUTTER, C. K. et al. Síntese de trans-resveratrol e controle de podridão em maçãs com uso de elicitores em pós-colheita. Pesquisa Agropecuária Brasileira, v.43, n.9, p.1097-1103, 2008.

TAO, Y.; ZHANG, L. Intensity prediction of typical aroma characters of cabernet sauvignon wine in Changli County (China). LWT - Food Science and Technology, v.43, p.1550-1556, 2010. https://doi.org/10.1016/j.Iwt.2010.06.003.

TAO, Y.S.; LIU, Y.; LI, H. Sensory characters of Cabernet Sauvignon dry red wine from Changli County (China). Food Chemistry, v. 114, n. 2, p. 565-569, 2008. https://doi.org/10.1016/j.foodchem.2008.09.087.

TREPTOW, T.C. Radiação UV-C em cultivares de uvas Niágara Branca e Trebbiano, Isabel e Carbenet Sauvignon. Dissertação (Mestrado). Universidade Federal de Santa Maria, p.83, 2012.

WELKE, J. Uso da microestração em fase sólida e da cromatografia gasosa monodimensional e bidimensional abrangente na caracterização de voláteis de vinhos gaúchos. Tese (Doutorado). Universidade Federal do Rio Grande do Sul, p.167, 2012.

$\mathrm{XI}$, Z. et al. Impact of cover crops in vineyard on the aroma compounds of Vitis vinifera L.cv Cabernet Sauvignon wine. Food Chemistry, v.127, n. 2, p. 516-522, 2011. https://doi.org/10.1016/j.foodchem.2011.01.033.

\section{Autores}

Simara Somacal ${ }^{1, *}$, Rodrigo Valvassori Gindri ${ }^{2}$, Fernanda Wouters Franco ${ }^{2}$, Sabrina Somacal $^{2}$, Cláudia Kaehler Sauter ${ }^{3}$, Fabrízio da Fonseca Barbosa ${ }^{4}$

1. Curso de Pós-Graduação Lato Sensu em Ciência dos Alimentos, Centro de Ciências Químicas, Farmacêuticas e de Alimentos, Universidade Federal de Pelotas, Campus Capão do Leão, Caixa Postal 354, CEP. 96010-900, Pelotas, RS, Brasil. Programa de Pós-Graduação em Ciência e Tecnologia dos Alimentos, Departamento de Ciência e Tecnologia dos Alimentos' Universidade Federal de Santa Maria, Campus Sede, CEP. 97105-900, Santa Maria, RS, Brasil.

2. Programa de Pós-Graduação em Ciência e Tecnologia dos Alimentos, Departamento de Ciência e Tecnologia dos Alimentos' Universidade Federal de Santa Maria, Campus Sede, CEP. 97105-900, Santa Maria, RS, Brasil.

3. Departamento de Ciência e Tecnologia dos Alimentos' Universidade Federal de Santa Maria, Campus Sede, CEP. 97105-900, Santa Maria, RS, Brasil. 
4. Centro de Ciências Químicas, Farmacêuticas e de Alimentos, Universidade Federal de Pelotas, Campus Capão do Leão, Caixa Postal 354, CEP. 96010-900, Pelotas, RS, Brasil.

* Autor para correspondência: $\underline{\text { s.somacal@gmail.com }}$ 